\title{
Chapter 5 \\ Cloud Material Handling Systems: Conceptual Model and Cloud-Based Scheduling of Handling Activities
}

\author{
Fabio Sgarbossa, Mirco Peron, and Giuseppe Fragapane
}

\begin{abstract}
Nowadays, the implementation of cloud manufacturing technologies epitomizes the avant-garde in production systems. This affects several aspects of the management of these production systems, in particular scheduling activities, due to the possibility provided by cloud manufacturing of having real-time information about the stages of a product life cycle and about the status of all services. However, so far, cloud manufacturing has mainly focused on machines, with limited interest in material handling systems. This shortfall has been addressed in this study, where a new material-handling paradigm, called Cloud Material Handling System (CMHS) and developed in the Logistics 4.0 Lab at NTNU (Norway), has been introduced. With CMHS, the scheduling of the Material Handling Modules (MHMs) can be optimized, increasing the flexibility and productivity of the overall manufacturing system. To achieve this, the integration of advanced industry 4.0 technologies such as Internet of Things (IoT), and in particular Indoor Positioning Technologies (IPT), Cloud Computing, Machine Learning (ML), and Artificial Intelligence (AI), is required. In fact, based on the relevant information provided on the cloud platform by IPT and IoT for each product, called Smart Object (SO) (position, physical characteristics and so on), an Intelligent Cognitive Engine (ICE) can use ML and AI to decide, in real time, which MHM is most suitable for carrying out the tasks required by these products based on a compatibility matrix, on their attributes, and on the defined scheduling procedure.
\end{abstract}

\subsection{Introduction}

In recent years, connectivity and exchange of information have played an important role in the world of manufacturing (Alcácer and Cruz-Machado 2019). Researchers

\footnotetext{
F. Sgarbossa $(\varangle) \cdot$ M. Peron · G. Fragapane

Department of Mechanical and Industrial Engineering Valgrinda, NTNU-Norwegian University of Science and Technology, Trondheim, Norway

e-mail: fabio.sgarbossa@ntnu.no; mirco.peron@ntnu.no; giuseppe.fragapane@ntnu.no
}

B. Sokolov et al. (eds.), Scheduling in Industry 4.0 and Cloud Manufacturing, International Series in Operations Research \& Management Science 289, https://doi.org/10.1007/978-3-030-43177-8_5 
and practitioners have focused their attention on new technologies (such as cyber physical systems [CPS], IoT, and cloud computing) that enable the integration of production with network connectivity (Xu 2012; Lee et al. 2018; Ivanov et al. 2016, 2019; Panetto et al. 2019; Fragapane et al. 2020; Battini et al. 2018). In this way, the traditional rigid and automated operational processes can evolve in fully connected and flexible systems, highly valuable in a market characterized by unpredictable changes. In fact, connecting different machines in production, material-handling systems in warehouses, and equipment in laboratories can form networks capable of dynamic reconfiguration and high flexibility, and can provide global feedback in order to achieve high efficiency.

Lately, the impact of COVID-19 outbreak on global industry has demonstrated the role of fully connected and flexible manufacturing system in contributing to the resilience of the supply chains. Companies with integrated manufacturing network seem to be better positioned at the crisis time and for the future recovery (Ivanov 2020; Ivanov and Das 2020; Ivanov and Dolgui 2020).

Most effort has been put toward the connectivity of different machines in production systems, with the establishment of cloud manufacturing. By connecting different machines with cloud services and managing them in a centralized way, the production systems can adapt, in real time, to new demands with increased flexibility (Liu et al. 2019). This new manufacturing paradigm influences several aspects, one of which is scheduling.

Scheduling is the process of arranging, controlling, and optimizing work or workloads. Therefore, in terms of allocating resources/services to tasks, monitoring, controlling, and optimizing task execution, the possibility provided by cloud manufacturing of having real-time information about all the stages of a product life cycle and about the status of all the services is of great impact. Considering the allocation of resources to different tasks, Liu et al. described the process as a sum of different sub-steps, at the end of which an optimized schedule is generated based on the real-time status information (availability, position, etc.) of the machines required to process an order (Liu et al. 2019; Dolgui et al. 2019).

As mentioned earlier, connectivity of different machines has been already developed, improving scheduling activities. However, a step forward toward the optimization of these new manufacturing scheduling procedures would be the implementation of cloud services for material-handling equipment. However, due to the complexity and variability of possible performed tasks, available resources, and equipment, the scheduling and planning of material-handling equipment has been mainly treated as separate from the manufacturing systems with which they operate.

Material handling is defined as the movement, storage, protection, and control of materials throughout the manufacturing and distribution process (including their consumption and disposal). It involves providing the right amount of the right material, in the right condition, at the right place, in the right position, in the right sequence, and for the right cost, by the right method(s). The main objective of material handling is to perform it safely, efficiently, at low cost, in a timely manner, accurately, and without damage. 
The typical structure of today's material-handling systems is a mix of different equipment with various levels of automation (Furmans and Gue 2018). It is still largely dominated by manual and mechanized systems, such as manual carts and industrial vehicles (i.e., pallet trucks, forklifts), in which humans still play an important decisional role. In other cases, automated solutions, such as Automated Guided Vehicles (AGVs) or Automated Storage and Retrieval Systems (AS/RS), are implemented with their own decentralized control systems. Since they are not connected to each other, a multilevel hierarchical control system is necessary to coordinate the different sub-systems and allow the products to be moved from one point to the next within the manufacturing system. Typically, decision-making processes such as scheduling are distributed over the levels of systems interacting with each other (such as the local PLCs, the material flow controller, the Warehouse Management System [WMS], Manufacturing Execution System [MES], and the Enterprise Resource Planning [ERP] system).

Decisions, processes, and activities in material-handling systems show great dependencies and should not be seen as isolated, independent procedures. Materials handling should be seen within a system context. The systems concept is particularly helpful because it identifies and analyzes the interrelation within a system. Blanchard and Fabrycky define a system as "a set of interrelated components working together with the common objective of fulfilling some designated need" (Blanchard and Fabrycky 1990). The efficient scheduling of the material handling equipment (MHE) has a strong effect on the productivity, profitability, and flexibility of the manufacturing systems, especially in a low-volume high-variety context (Zangaro et al. 2019; Zennaro et al. 2019a, b). Some examples are a machine waiting for the product to process since the forklift driver is not available, or a machine being blocked because the unit loads in the unloading station are still waiting to be transported to the next production phase. The availability in recent years of industry 4.0 technologies, such as IPT as part of IoT, motion tracking and control, and cloud computing is making MHE one of the most feasible solutions for increasing the flexibility of manufacturing systems.

By extending the definition of cloud manufacturing to handling activities, a new kind of paradigm, called a Cloud Material Handling System (CMHS), has been introduced and developed by the authors in the Logistics 4.0 Laboratory at the Norwegian University of Science and Technology. This is Norway's first logistics laboratory that merges digital technologies with traditional production and logistics systems, enabling researchers, practitioners, engineers, pioneers, students, and other enthusiasts to come together and collaborate on common ground.

As described previously, the typical aim of cloud manufacturing is to deliver on-demand manufacturing services to customers based on orders received via the Internet. With the CMHS, this aim can be adapted to the efficient management and scheduling of MHE. Based on the same concept as cloud manufacturing, the CMHS has the scope to satisfy consumers' requests (handling of unit loads) through the available resources (MHE) in a cloud environment, reducing the complexity of a multilevel hierarchical control system and increasing the overall flexibility and productivity of the manufacturing system. The CMHS has been developed mostly 
for applications within a single factory, but it can be also extended to a multi-factory environment where the logistics activities are, for example, external transportation.

For this purpose, the CMHS needs information that is not typically collected and used in cloud manufacturing. According to the definition of material handling (movement, storage, protection, and control), the most important information required from the system is the real-time locations of products and MHE. This lack can be filled by implementing IPT, allowing real-time localization of the products/unit loads and Material Handling Equipment in a cloud platform. IPT is in fact a technology that continuously determines in real time the position of something or someone in a physical space (Hightower and Borriello 2001b). As part of IoT, according to Gu et al. (2009), an IPT can provide different kinds of data, including position, travel path, time, speed, and required activities. Depending on the applications, there are several different kinds of IPT. They will be described in the next section, focusing on their characteristics and on their pros and cons. In Sect. 5.3, the CMHS will be introduced and described, while in Sect. 5.4, the characteristics of scheduling in the CMHS will be described and compared to the traditional method. Finally, conclusions, future research, and perspectives will be provided in Sect. 5.5.

\subsection{Indoor Positioning Technologies}

IPT, as part of the IoT, is defined as the sub-system that permits a mobile device to determine its position and that renders this position available for position-based services (Gu et al. 2009). These position-based services can bring benefits in several environments, such as hospitals, where the position of the equipment needs to be known to efficiently use the medical resources, supermarkets, where the customers want to know the fastest path to reach the desired products, and large museums, where tourists are interested in knowing the location of the artworks they are interested in Tesoriero et al. (2008). In the last few years, IPT has gained interest also in the industrial field (Zuin et al. 2018). In particular, material-handling processes are those that can benefit the most from this system. In fact, given the need for handling huge volumes of products with very short lead times, a material-handling system has to eliminate all the inefficiencies, such as delays in the search for the required product in the warehouse, errors in the storage or in the picking of an item, and waste of time during the travelling of carts and operators.

As stated by Curran et al. (2011), there are several available technologies to identify, in real time, the location and flow of material depending on the required performances. There is not a single "best solution" that is suitable for each scenario. Therefore, in recent years, different types of IPTs have been introduced depending on the desired level of accuracy, coverage area, robustness, scalability, cost, and complexity (Gu et al. 2009). They can use one or several positioning technologies (i.e., triangulation, fingerprinting, proximity, and vision analysis (Kaemarungsi and Krishnamurthy 2004; Hightower and Borriello 2001)), but the majority leverage the triangulation method, where once the coordinates $X$ and $Y$ of the three reference 
elements $A, B$, and $C$ are known, the position can be calculated by using either the length or the directions of at least three vectors from the respective reference points (Gu et al. 2009).

However, the classification of these IPTs is not usually based on the positioning technology used. Instead, it is usually based on either network features (Deak et al. 2012) or on their hardware requirements (Liang et al. 2013). Another classification can be based on the main technology used to determine the location, which may include infrared (IR) signals, ultrasound waves, vision-based analysis, and radio frequency.

Infrared positioning systems. This is one of the most common positioning systems since IR emitters are small and lightweight. The system architecture is simple, and it performs positioning estimation in a very accurate way (with an accuracy of several $\mathrm{mm}$ ). It was used by Pinto et al. for the localization of small mobile robots, and they reported high accuracies $\left(0.06 \mathrm{~m}\right.$ and $\left.7^{\circ}\right)$ and fast responsiveness in the localization (less than $40 \mathrm{~ms}$ ) (Pinto et al. 2015). Similar results were reported in Zhang et al. (2010), where an accuracy of $3 \mathrm{~mm}$ and a responsiveness of $3 \mathrm{~ms}$ were reported. However, they also reported a limited coverage area $(7 \mathrm{~m})$. In fact, one of the main limitations is related to the application environment of this system, as it requires the absence of interferences and obstacles. Nevertheless, this can also be an advantage; the inability of the IR beams to penetrate the walls ensures that it is possible to limit the signal inside a specific room (Mainetti et al. 2014). Other limitations are the high cost of the hardware, the short-range signal transmission between devices, and the interference from florescent light and sunlight (Fernando et al. 2003).

Ultrasound positioning systems: This system uses ultrasound signals to measure the distance of a mobile target from a fixed-point receiver. Despite its low cost, the diffusion of this IPT is hampered by the low accuracy (several $\mathrm{cm}$ ) and the short range (from 2 to $10 \mathrm{~m}$ ). These drawbacks have been shown to be overcome by using a large number of transmitters on the ceiling (Woodman and Harle 2010). However, in doing so, one of the main advantages of this IPT (low cost) would be offset. Both studies reported the system to be highly susceptible to noise sources, which affect its reliability and accuracy.

Vision-based positioning systems: This system is based on the use of fixed or mobile cameras that can cover a large area at a low installation cost. The tracked person and/or device does not need to wear or carry any device. It has been successfully used as a tracking system for rolled milled plates with an accuracy of $0.5 \mathrm{~m}$ (Tratnig et al. 2007). However, some inaccuracies were reported, especially in a dynamic environment and in environments characterized by many interference sources. These systems are, in fact, preferable for quality control and inventorylevel assessments (Wu et al. 2009). In addition, the difficulty in tracking several persons and/or devices has also limited its use (Zuin et al. 2018).

Radio frequency position systems: There are several systems that use radio waves, such as Wi-Fi, Radio Frequency Identification (RFID), and Ultra-Wide Band (UWB). The Wi-Fi system is often used to monitor the movements of mobile 
devices due to its low cost. In fact, Wi-Fi is widely spread inside the facilities and thus extra software are not required. Accuracy is quite low (between 20 and 40 $\mathrm{m}$ ) due to problems of refraction, scattering, and multi-path fading in the indoor propagation of Wi-Fi signals. This can be improved by increasing the number of access points and adding wireless routers (Chen et al. 2014), reaching an accuracy of $2 \mathrm{~m}$ (Han and He 2018), or by using particular filters, such as Unscented Kalman Filter (Khan et al. 2017). RFID is a means of storing and retrieving data through radio waves to an RF-compatible integrated circuit (Ni et al. 2003). These systems can cover large distances since they do not require line-of-sight (i.e., they can easily travel through walls and human bodies). Moreover, RFID leverages small and light tags and this allows for a unique identification of equipment and persons. However, this system needs several infrastructure components in the working area and they suffer from multi-path distortion of radio signals reflected by walls and obstacles ( $\mathrm{Gu}$ et al. 2009). UWB technology uses pulses with a very short duration (less than $1 \mathrm{~ns}$ ) to overcome the limitation of the multi-path distortion of radio signals that affects RFID systems. In addition, the use of multiple bands of frequencies simultaneously (each transmitting its own signal, as opposed to RFID, which uses just a single portion of the frequency spectrum) overcomes RFID's line-of-sight requirement and thus increases accuracy. That was shown by Regattieri and Santarelli, who tested a UWB system in different industrial environments and reported an accuracy of $1 \mathrm{~m}$ (Regattieri and Santarelli 2013). These results agreed with those reported in Zuin et al. (2018), who found that $70 \%$ of measurements of a moving target showed the gap between the tracked position and the real one as lower than $0.40 \mathrm{~m}$.

By using IPT, it is thus possible to have real-time information about products/operators/MHE location in an easy and fast way, representing a prerogative for the development of the CMHS, since it requires the real-time location of all the elements of the system.

\subsection{Cloud Material Handling System}

Uber is revolutionizing the world of urban logistics (i.e., people mobility and product delivery) by providing peer-to-peer ridesharing, ride service hailing, food delivery, and a bicycle sharing system. Its success is based on the cloud storage and smart use of huge amounts of data regarding the real-time locations of consumers and cars/bicycles/scooters.

Using Uber is very simple both for consumers and drivers. The phone app helps the users in setting the pickup location (using GPS), setting the destination, and requesting a car. It also supports the drivers in managing requests and receiving payments. However, there is a huge amount of data processed in a cloud platform, even when the driver has no passengers or products out for delivery. All this data is stored and leveraged to predict supply and demand, as well as for setting fares and gathering information about issues such as bottlenecks, traffic jams, and shortcuts. 


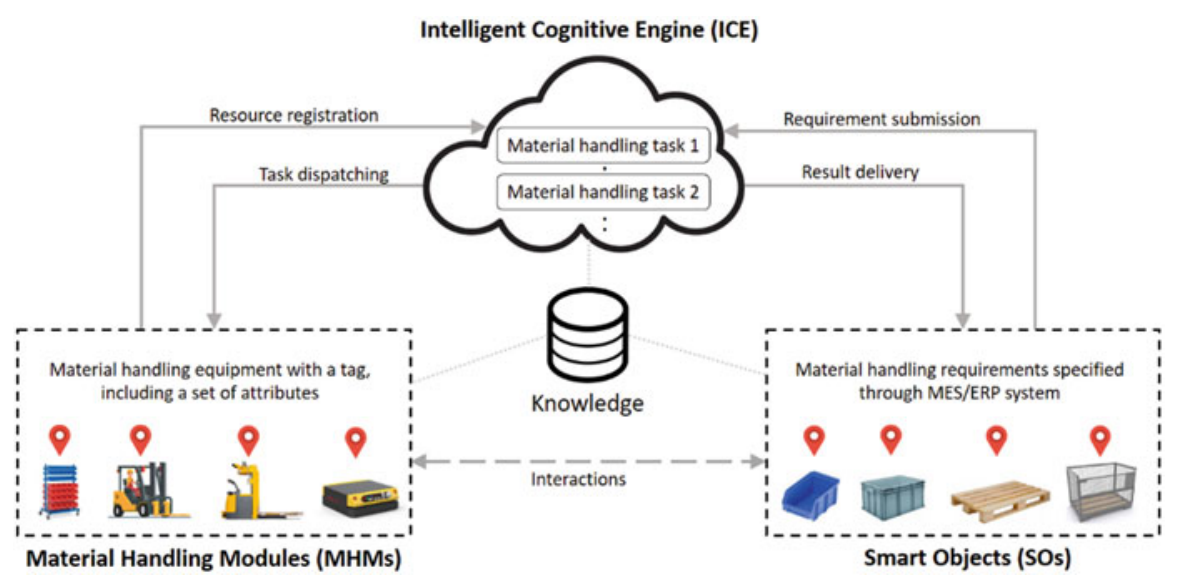

Fig. 5.1 Operation model of Cloud Material Handling System

The concept behind the CMHS developed by the authors at the Logistics 4.0 Laboratory at NTNU is similar to the taxi-hailing web app. In this case, the "consumers" are the unit loads that require a specific service from the system (typically to be transported from one point to another), while the "cars" and "drivers" are the MHE (forklifts, manual trolleys, conveyors, etc.) with different capabilities (capacity, cost, speed, time, service level, etc.). The cloud platform has a cognitive engine able to dynamically assign the requests to the available resources based on knowledge gained over time.

Similarly to cloud manufacturing (Liu et al. 2019; Zhang et al. 2014), the operation model of the Cloud Material Handling System has a direct effect on the characteristics of the scheduling. Figure 5.1 depicts the operation model developed by the authors at the base of the CMHS. Adapting the concept introduced by Zhang et al. (2014), it consists of three categories of stakeholders: SOs, Material Handling Modules (MHMs), and ICE, sharing a common knowledge of the system.

SOs: These are the unit loads that require handling by the MHE (Furmans and Gue 2018). They are uniquely identified through a tag containing a dynamic set of attributes, such as material flow or process of the object, the current and next step of the process, or its physical characteristics (size, weight, fragility, etc.). The tag is localized through one of the IPTs described in the previous section (Fig. 5.2).

Material handling modules (MHMs): These have the capabilities to perform one or more physical functions related to handling and storing, as required by the SOs. MHMs are typical MHE, such as forklifts, conveyors, cranes, trolleys and carts, AGVs, AMRs, shelving units, storage racks, or ASR/RS (Furmans and Gue 2018). They can be purely manual or mechanized equipment, where the operators still play an important role, like driving, loading, or unloading, but also fully automated equipment with automatized functions. Like the SOs, they are classified in the CMHS with a tag including a set of attributes, such as 


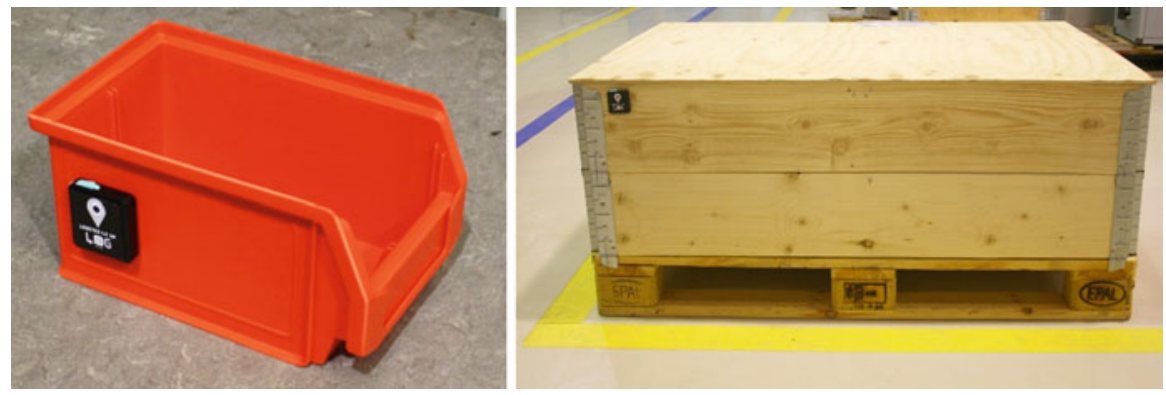

Fig. 5.2 Examples of SOs

dimensions, capacity, autonomy, automation level, and cost. They also have a set of functions they are able to perform. According to Furmans and Gue (2018), four classes can be identified: holder (storing an SO, such as shelves and racks); mover (moving an SO, such as forklifts); picker-placer (picking and placing an SO, such as human pickers or robot arms); and unitized-separator (putting together more SOs, such as a palletizer, or creating more SOs from an initial single one, such as a depalletizer). They are connected to the ICE, communicating their locations in real time thanks to IPT. Their availability and lists of attributes/functions are shared with the other stakeholders in the cloud platform, through which they receive jobs. In case they require the human to be active, such as manual trolleys or traditional pallet jacks or forklifts, the interaction with the ICE is based on the use of industrial smartphones or devices, through which the operators receive the scheduled assigned tasks after processing in the ICE (Fig. 5.3).

ICE: This is responsible for the management of the cloud platform where all the information is collected and elaborated (Greis et al. 2019). It receives and stores all the information about the available MHMs and their attributes and functions. These characteristics are matched with the handling requirements sent by the active SOs through an MHM-SO compatibility matrix. The ICE is an AI-based computing system, and thanks to several ML algorithms, it can predict how the global CMHS performs in different situations. In the ICE, the models, rules, and algorithms for scheduling are implemented. Their performance, such as the percentage of loaded travel time, the percentage of added value time, or service level, is assessed in real-time in order to be improved over time through what-if scenarios simulation (Fig. 5.4).

\subsection{Scheduling in Cloud Material Handling System}

The real-time localization of the SOs and MHMs due to the IPT implementation, and the sharing of their attributes/functions along with positions are enabling a 

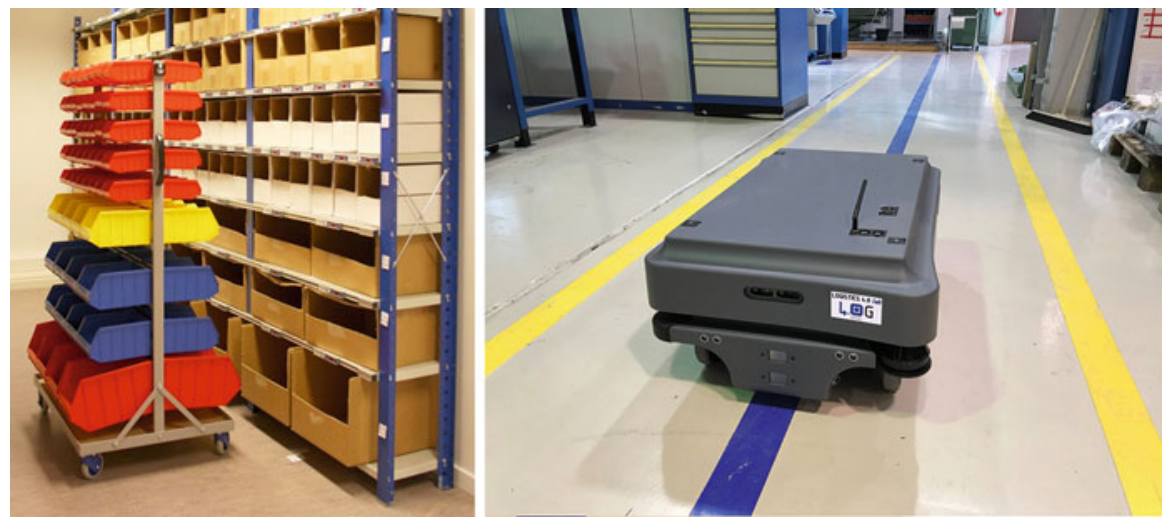

Fig. 5.3 Examples of MHMs

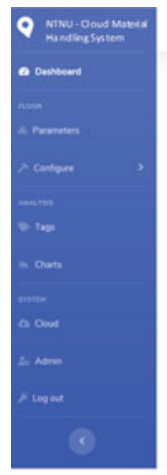

Dashboard - Intelligent Cognitive Engine

\begin{tabular}{|c|c|c|}
\hline \multicolumn{3}{|l|}{ Connection } \\
\hline \multicolumn{3}{|c|}{ Sutue Comented 8} \\
\hline \multicolumn{3}{|l|}{ Discumed } \\
\hline \multicolumn{3}{|c|}{ Smart Objects } \\
\hline $50 \mathrm{Tag}$ & Status & Location \\
\hline 0100251324 & wating & butfero7 \\
\hline 0100542159 & moving & MaMMOO2 \\
\hline 0258700032 & stocking & MHMOO4 \\
\hline 0580015200 & in proveress & mechine 003 \\
\hline 0622501120 & wating & butter21 \\
\hline 0658114203 & wating & buffer11 \\
\hline
\end{tabular}
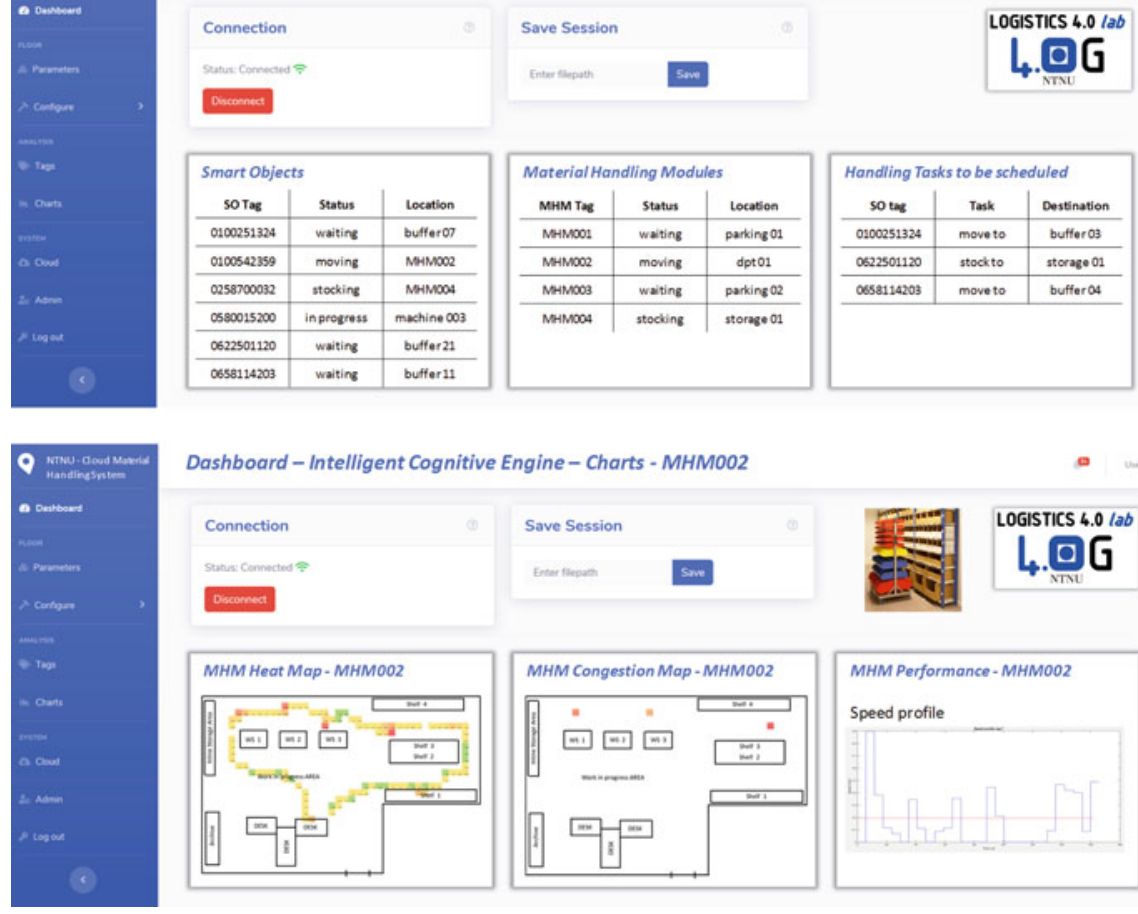

Dashboard - Intelligent Cognitive Engine - Charts - MHMOO2
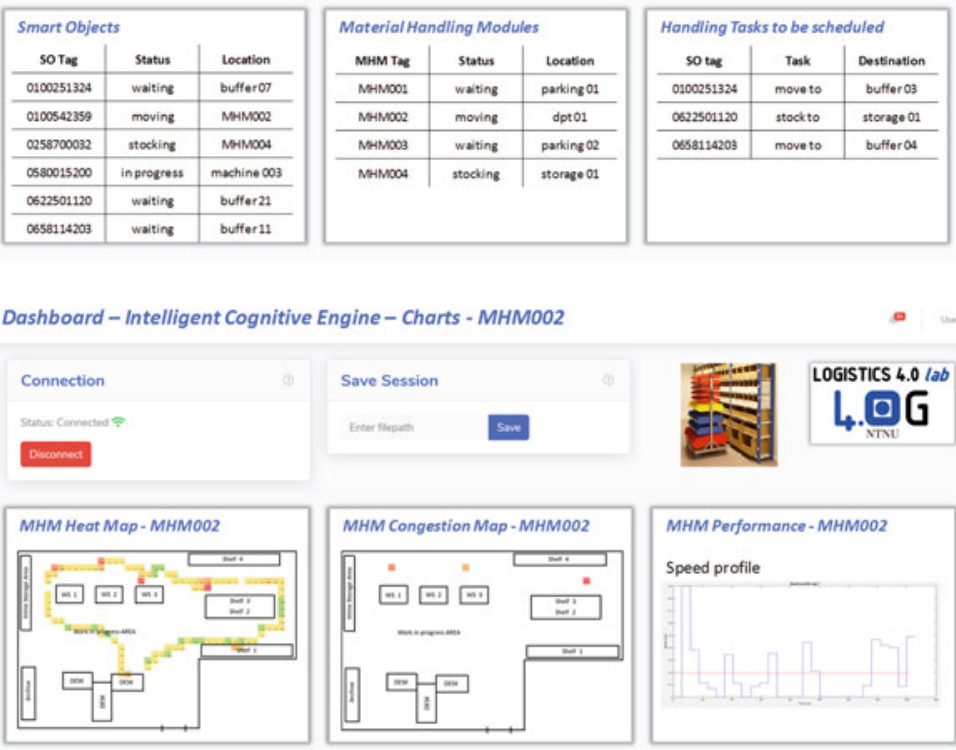

Fig. 5.4 Screenshots of the ICE dashboard

new way of scheduling and control of all the components in the system. In this section, the procedure for assignment of the required jobs by SOs to the different available MHMs is described, and the characteristics of the scheduling in CMHS are 
presented in comparison to the scheduling in a traditional environment. The industrial implications and advantages in its application will be illustrated and finally some research opportunities are discussed.

\subsubsection{Procedure of Scheduling}

There are three different phases for the scheduling in CHMS: order/task release, scheduling and delivery, and performance assessment.

Order/task release: The scheduling process begins when SOs send their requirement of a specific service to ICE. The specific service may be the need for the SOs to be stored, moved, transferred, picked, placed, unitized, separated, and/or a combination of these needs. It can be triggered manually by the operator of the machine/MHM where the SO is produced/moved. IPTs can automatize this step of the process. Specific areas can be defined in the cloud platform; when the location of the SOs (using IPT) is within one of these areas, it could be linked to the claiming of service from MHMs. Other areas could be used as destination of the service; when the SOs are moved into those areas, it means that the required tasks have been performed. These tasks are defined a priori thanks to the connection to the ERP system and material flows/production processes.

Scheduling: Every SO is uniquely identified by a tag containing all the relevant information (characteristics such as size, weight, the next step of the process). Based on these characteristics, the ICE can decide the most suitable type of MHM, leveraging the MHM-SO compatibility matrix. Once the type of MHM that fulfils the handling requirements of the SO has been defined, the required MHM is then found among those available according to the pre-defined scheduling procedure (i.e., the closest MHM, the fastest MHM, the cheapest). AI and scheduling algorithms derived from those developed for cloud manufacturing will support this phase of the process in the case of more complex environments.

Delivery and performance assessment: Once the MHM has completed its task, data such as the average speed, the path, congestion phenomena, percentage of added value, time, and other performances can be obtained by ICE through the extrapolation of the data from the tag associated with the SO under consideration. In this way, the ICE can use the acquired information in order to optimize the future tasks using the acquired data as input in its ML algorithms.

\subsubsection{Characteristics of Scheduling in CMHS}

The scheduling of material-handling tasks is a decision-making process at an operational level. In today's manufacturing and services industries, the coordination of these tasks is mainly hierarchical in structure, with centralized or decentralized 
control (Scholz-Reiter and Freitag 2007). Several decision stages are thereby, to a different degree, interconnected and automated. Low degree of such decisions have led to fixed assignment using either simple scheduling rules as FIFO (First in first out) or EDD (Earliest Due Date), weighted priority rules, or heuristics scheduling rules. The complexity of the material flow and the push toward increased automation have led to standardized multilevel control architectures and information flow for material-handling systems (Furmans and Gue 2018). The standards contain the tasks to be performed on each level and the communication protocols between the levels. The decision-making process is normally distributed over several system levels: PLC, ERP, MES, process, and machine control. Within a company using material-handling systems, there can typically be found between five and eight levels of systems interacting with each other. Multilevel design and scheduling is often motivated by a company's low capability for real-time information sharing. The introduction of CMHS changes the characteristics of scheduling as follows:

Knowledge-sharing-based scheduling: The introduction of the cloud platform and its ICE modified the way of doing scheduling. In the CMHS, all the equipment are at the same level as the handled products. This allows for real-time adaptability of the MHMs to the current requirements of the systems with which they operate. Each module and object are individual stakeholders, autonomous decision-makers, and interest-independent entities (Liu et al. 2019).

Many MHMs to many jobs scheduling: The sharing of information about locations and attributes of MHMs and SOs enables the distribution of multiple jobs to multiple integrated resources. In traditional MHS, each job is managed individually from one single resource, or it is treated as a sequence of elementary jobs to be executed by single resources.

Dynamic and complex scheduling: Scheduling in CMHS is more complex due to the two characteristics described previously. In this case, the application of AI and ML can support the scheduling, learning from previous experience, and assessing the performance of each single module of the system. Rules, models, and algorithms are developed based on the huge amount of data collected over time.

The CMHS allows for more efficient scheduling since all MHMs are shared among all the SOs requiring a handling activity (compared to traditional MHS where, for example, equipment is limited to a specific area or specific material flow and unit loads). Simple examples of its benefits are MHMs idle while no SOs require them in the area can move to another area to serve other SOs and share their availability with the rest of the CMHS; delay in handling an SO due to over-utilizing of MHMs can be reduced using the other similar equipment available in other areas of the systems. The basic concept is based on a shared equipment, which allows reducing the total number of required modules as compared to a case with fixed assignments. Therefore, the average usage of the MHMs is higher, as is the service level and responsiveness of the system. Moreover, the introduction of AI and ML in the ICE allows for increased efficiency in every scheduled task thanks to the learning effect based on the huge amount of data collected over time. The big data available (about the travelled path, delivery time, travel time, service level, etc.) can provide useful 
feedback on the efficiency of the scheduling, enabling the AI and ML to improve future scheduling.

Scheduling in CMHS still faces many challenges. For example, it is still very important to consider how to manage the different interests and objectives of the different MHMs and SOs in the system. The scheduling could be based on a market mechanism for coordinating the activities of the MHMs that pursue their own interest. The market model could be used for solving dynamic job processing and scheduling problems. Conflicts between individual requirements could be resolved by negotiating and bargaining on simple terms such tasks, due dates, and prices (Márkus et al. 1996). New game theory, negotiation methodology, cooperation, and coordination among the MHMs are just some examples of new research areas for more efficient scheduling. Another example is how to define the optimal level of control between a fully centralized system with ICE as the main actor and a fully decentralized/holonic system where the MHMs and SOs act as individual intelligent agents. Several control models from other fields, such as cloud manufacturing, can be applied to support the scheduling.

\subsection{Conclusions}

Sharing real-time information on MHE and unit loads has enabled and changed the decision-making process for scheduling of material-handling activities from a hierarchically structured and centralized control system to a cloud-based one. With the introduction of the CMHS, the scheduling of material-handling activities can be optimized, leading to increasing flexibility and productivity of the overall manufacturing system. Current literature does not specify how production systems should be adapted from a material-handling perspective to enable cloud manufacturing. We contribute to the existing literature by introducing a conceptual model of CMHS and application at NTNU's Logistics 4.0 laboratory, reflecting a manufacturing environment. The current state of the IPTs and CMHS has been described. A special focus has been given to scheduling, highlighting the differences and benefits for future material-handling systems. Future research should investigate how AI and ML can further improve scheduling and consecutive activities of routing and dispatching, which have not been investigated until now.

\section{References}

Alcácer, V., \& Cruz-Machado, V. (2019). Scanning the Industry 4.0: A literature review on technologies for manufacturing systems. Engineering Science and Technology, an International Journal, 22(3), 899-919. https://doi.org/10.1016/J.JESTCH.2019.01.006.

Battini, D., Calzavara, M., Persona, A., Sgarbossa, F., Visentin, V., \& Zennaro, I. (2018). Integrating mocap system and immersive reality for efficient human-centred workstation design. IFACPapersOnLine, 51(11), 188-193. 
Blanchard, B. S., \& Fabrycky, W. J. (1990). Systems engineering and analysis (Pearson International Edition) (4th ed.). Englewood Cliffs, NJ: Prentice Hall. Retrieved from http://sutlib2.sut.ac.th/ sut_contents/H104135.pdf.

Chen, L.-H., Wu, E. H.-K., Jin, M.-H., \& Chen, G.-H. (2014). Intelligent fusion of Wi-Fi and inertial sensor-based positioning systems for indoor pedestrian navigation. IEEE Sensors Journal, 14(11), 4034-4042. https://doi.org/10.1109/JSEN.2014.2330573.

Curran, K., Furey, E., Lunney, T., Santos, J., Woods, D., \& McCaughey, A. (2011). An evaluation of indoor location determination technologies. Journal of Location Based Services, 5(2), 61-78. https://doi.org/10.1080/17489725.2011.562927.

Deak, G., Curran, K., \& Condell, J. (2012). A survey of active and passive indoor localisation systems. Computer Communications, 35(16), 1939-1954. https://doi.org/10.1016/ J.COMCOM.2012.06.004.

Dolgui, A., Ivanov, D., Sethi, S. P., \& Sokolov, B. (2019). Scheduling in production, supply chain and Industry 4.0 systems by optimal control. International Journal of Production Research, 57(2), 411-432.

Fernando, X. N., Krishnan, S., Sun, H., \& Kazemi-Moud, K. (2003). Adaptive denoising at infrared wireless receivers. In B. F. Andresen \& G. F. Fulop (Vol. 5074, p. 199). International Society for Optics and Photonics. https://www.spiedigitallibrary.org/conference-proceedings-of-spie/5074/ 0000/Adaptive-denoising-at-infrared-wireless-receivers/10.1117/12.486354.short

Fragapane, G., Ivanov, D., Peron, M., Sgarbossa, F., \& Strandhagen, J. O. (2020). Increasing flexibility and productivity in industry 4.0 production networks with autonomous mobile robots and smart intralogistics. Annals of operations research, 1-19.

Furmans, K., \& Gue, K. (2018). A framework for modeling material handling with decentralized control. In Progress in Material Handling Research, January. Retrieved from https:// digitalcommons.georgiasouthern.edu/pmhr_2018/23.

Greis, N. P., Nogueira, M. L., Schmitz, T., \& Dillon, M. (2019). Manufacturing-Uber: Intelligent operator assignment in a connected factory. In Ninth IFAC Conference on Manufacturing Modelling, Management and Control, Berlin. Retrieved from https://ifac.papercept.net/ conferences/conferences/MIM19/program/MIM19_ContentListWeb_3.html.

Gu, Y., Lo, A., \& Niemegeers, I. (2009). A survey of indoor positioning systems for wireless personal networks. IEEE Communications Surveys \& Tutorials, 11(1), 13-32. https://doi.org/ 10.1109/SURV.2009.090103.

Han, X., \& He, Z. 2018. A wireless fingerprint location method based on target tracking. In 2018 12th International Symposium on Antennas, Propagation and EM Theory (ISAPE) (pp. 1-4). IEEE. https://doi.org/10.1109/ISAPE.2018.8634177.

Hightower, J., \& Borriello, G. (2001). Location sensing techniques. Retrieved from http:// kom.aau.dk/group/05gr999/reference_material/locationtechniques/hightower2001techniques. pdf.

Ivanov, D. (2020). Predicting the impacts of epidemic outbreaks on global supply chains: A simulation-based analysis on the coronavirus outbreak (COVID-19/SARS-CoV-2) case. Transportation Research Part E: Logistics and Transportation Review, 136, 101922.

Ivanov D., Dolgui A. (2020). Viability of intertwined supply networks: Extending the supply chain resilience an-gles towards survivability. A position paper motivated by COVID-19 outbreak. Accepted for Publication in International Journal of Production Research.

Ivanov D., Das A. (2020). Coronavirus (COVID-19 / SARS-CoV-2) and supply chain resilience: A research note. Accepted for Publication in International Journal of Integrated Supply Management.

Ivanov, D., Sokolov, B., Dolgui, A., Werner, F., \& Ivanova, M. (2016). A dynamic model and an algorithm for short-term supply chain scheduling in the smart factory Industry 4.0. International Journal of Production Research, 54(2), 386-402.

Ivanov, D., Dolgui, A., \& Sokolov, B. (2019). The impact of digital technology and Industry 4.0 on the ripple effect and supply chain risk analytics. International Journal of Production Research, 57(3), 829-846. 
Kaemarungsi, K., \& Krishnamurthy, P. (2004). Properties of indoor received signal strength for WLAN location fingerprinting. In The First Annual International Conference on Mobile and Ubiquitous Systems: Networking and Services, 2004. MOBIQUITOUS 2004 (pp. 14-23). IEEE. https://doi.org/10.1109/MOBIQ.2004.1331706.

Khan, M., Kai, Y. D., \& Gul, H. U. (2017). Indoor Wi-Fi positioning algorithm based on combination of location fingerprint and unscented Kalman filter. In 2017 14th International Bhurban Conference on Applied Sciences and Technology (IBCAST) (pp. 693-698). IEEE. https://doi.org/10.1109/IBCAST.2017.7868127.

Lee, C. K. M., Yaqiong, L., Ng, K. K. H., Ho, W., \& Choy, K. L. (2018). Design and application of internet of things-based warehouse management system for smart logistics. International Journal of Production Research, 56(8), 2753-2768. https://doi.org/10.1080/ 00207543.2017.1394592.

Liang, J. Z., Corso, N., Turner, E., \& Zakhor, A. (2013). Image based localization in indoor environments. In 2013 Fourth International Conference on Computing for Geospatial Research and Application (pp. 70-75). IEEE. https://doi.org/10.1109/COMGEO.2013.11.

Liu, Y., Wang, L., Wang, X. V., Xu, X., \& Lin, Z. (2019). Scheduling in cloud manufacturing: Stateof-the-art and research challenges. International Journal of Production Research, 57(15-16), 4854-4879. https://doi.org/10.1080/00207543.2018.1449978.

Mainetti, L., Patrono, L., \& Sergi, I. (2014). A survey on indoor positioning systems. In 2014 22nd International Conference on Software, Telecommunications and Computer Networks (SoftCOM) (pp. 111-120). IEEE. https://doi.org/10.1109/SOFTCOM.2014.7039067.

Márkus, A., Kis Váncza, T., \& Monostori, L. (1996). A market approach to Holonic manufacturing. CIRP Annals, 45(1), 433-436. https://doi.org/10.1016/S0007-8506(07)63096-0.

Ni, L. M., Liu, Y., Lau, Y. C., \& Patil, A. P. (2003). LANDMARC: Indoor location sensing using active RFID. In Proceedings of the First IEEE International Conference on Pervasive Computing and Communications, 2003 (PerCom 2003) (pp. 407-415). IEEE Computer Society. https://doi.org/10.1109/PERCOM.2003.1192765.

Panetto, H., Iung, B., Ivanov, D., Weichhart, G., \& Wang, X. (2019). Challenges for the cyberphysical manufacturing enterprises of the future. Annual Reviews in Control, 47, 200-213.

Pinto, A. M., Moreira, A. P., \& Costa, P. G. (2015). A localization method based on map-matching and particle swarm optimization. Journal of Intelligent \& Robotic Systems, 77(2), 313-326. https://doi.org/10.1007/s10846-013-0009-2.

Regattieri, A., \& Santarelli, G. (2013). Manufacturing logistics and packaging management using RFID. In Radio frequency identification from system to applications. Rijeka: InTech. https:// doi.org/10.5772/53890.

Scholz-Reiter, B., \& Freitag, M. (2007). Autonomous processes in assembly systems. CIRP Annals, 56(2), 712-729. https://doi.org/10.1016/J.CIRP.2007.10.002.

Tesoriero, R., Gallud, J., Lozano, M., \& Penichet, V. (2008). Using active and passive RFID technology to support indoor location-aware systems. IEEE Transactions on Consumer Electronics, 54(2), 578-583. https://doi.org/10.1109/TCE.2008.4560133.

Tratnig, M., Reisinger, J., \& Hlobil, H. (2007). A vision-based material tracking system for heavy plate rolling mills. In D. Fofi \& F. Meriaudeau (Eds.) (Vol. 6356, p. 63560B). International Society for Optics and Photonics. https://www.spiedigitallibrary.org/conferenceproceedings-of-spie/6356/63560B/A-vision-based-material-tracking-system-for-heavy-platerolling/10.1117/12.736728.short?SSO=1\&tab=ArticleLink.

Woodman, O. J., \& Harle, R. K. 2010. Concurrent scheduling in the active bat location system. In 2010 Eighth IEEE International Conference on Pervasive Computing and Communications Workshops (PERCOM Workshops) (pp. 431-437). IEEE. https://doi.org/ 10.1109/PERCOMW.2010.5470631.

Wu, C. H., Ip, W. H., \& Chan, C. Y. (2009). Real-time distributed vision-based network system for logistics applications. International Journal of Intelligent Systems Technologies and Applications, 6(3/4), 309. https://doi.org/10.1504/IJISTA.2009.024260.

$\mathrm{Xu}, \mathrm{X}$. (2012). From cloud computing to cloud manufacturing. Robotics and Computer-Integrated Manufacturing, 28(1), 75-86. https://doi.org/10.1016/J.RCIM.2011.07.002. 
Zangaro, F., Finco, S., Battini, D., \& Zennaro, I. (2019). An optimization model for the storage assignment of the reference under ergonomics constraints. In Proceedings of the Summer School Francesco Turco, September 11-13, 2019, Brescia, IT.

Zennaro, I., Finco, S., Battini, D., \& Persona, A. (2019a). Big size highly customised product manufacturing systems: A literature review and future research agenda. International Journal of Production Research, 57(15-16), 5362-5385.

Zennaro, I., Calzavara, M., Finco, S., Battini, D., \& Persona, A. (2019b). A new part feeding model for big size and highly customized product assembly systems. In 25th International Conference on Production Research Manufacturing Innovation: Cyber Physical Manufacturing, August 9-15, 2019, Chicago, US.

Zhang, D., Xia, F., Yang, Z., Yao, L., \& Zhao, W. 2010. Localization technologies for indoor human tracking. In 2010 Fifth International Conference on Future Information Technology (pp. 1-6). IEEE. https://doi.org/10.1109/FUTURETECH.2010.5482731.

Zhang, L., Luo, Y., Tao, F., Li, B. H., Ren, L., Zhang, X., Guo, H., Cheng, Y., Hu, A., \& Liu, Y. (2014). Cloud manufacturing: A new manufacturing paradigm. Enterprise Information Systems, 8(2), 167-187. https://doi.org/10.1080/17517575.2012.683812.

Zuin, S., Calzavara, M., Sgarbossa, F., \& Persona, A. (2018). Ultra-wide band indoor positioning system: Analysis and testing of an IPS technology. IFAC-PapersOnLine, 51(11), 1488-1492. https://doi.org/10.1016/j.ifacol.2018.08.292. 